\title{
A Novel Approach for Classification of Underactuated Mechanism in Myoelectric Hand
}

\author{
M. Mariappan ${ }^{1, *}$, Sh. M. M. Jan ${ }^{1}$, M. Iftikhar ${ }^{2}$ \\ ${ }^{1}$ School of Engineering \& Information Technology, Universiti Malaysia Sabah, 88400, Kota Kinabalu, Malaysia \\ ${ }^{2}$ School of Medicine, Universiti Malaysia Sabah, 88400, Kota Kinabalu, Malaysia
}

\begin{abstract}
Concept of underactuation makes it possible to create such robotic hands that automatically adapt to the shape of the grasped object without using a complex control system or a large number of actuators. In underactuated hand prostheses, depending upon control strategy used, one can get more and more degree of freedoms (DOFs) to make its prosthetic device more versatile and easy to control. In spite of all the great work done by the researchers on underactuated prosthetic hands, no one has given any relationship between the number of motors (or actuators) and the number of degree of freedoms (DOFs). This novel concept named as "Coefficient of UAM" (CoUAM) is "the ratio between numbers of motors to the number of DOFs". By reviewing various known studies, CoUAM of randomly selected prosthetic hands is calculated separately and analyzed merits/demerits, cost, weight, appearance, ease of controllability and their functionality to make this approach more objective and useful for the future researchers. Hence target of this paper is to assess a Coefficient of UAM (CoUAM) of different types of prostheses available and then classify them into red, green and yellow zones.
\end{abstract}

Keywords Prosthetic Hand, Underactuated Mechanism, Degree Of Freedom, Myoelectric Prosthesis, Mitten Prosthesis

\section{Introduction}

In spite of the availability of various options: starting from mitten prosthesis to myoelectric hand, the rejection rate for using the prosthetic device is very high due to the heavy weight of prosthetic hands, the low functionality of prosthetic hands (resulted in single and unstable grasp caused by few finger's DOFs), the unnatural movements (caused by the limited DOFs) and the appearance of prosthetic hands is far from human hand. By seeing the negligence of users towards the prosthetic device different approaches has been carried out to fill a gap between prosthetic hand and the real human hand as in[1]. Due to all or any of these reasons only $30 \%$ to $50 \%$ handicap persons used their prosthetic hands once in a while[2].

Traumatic amputations including industrial accidents are leading causes of hand amputations in human being. Various efforts in the past few years are being carried out to make myoelectric hand as close as possible to the real human hand. Myoelectric prostheses deal with the concept, implementation and clinical application of utilizing inherent electrical signals with-in normally innervated residual muscles under voluntary control of an upper limb amputee. In myoelectric

* Corresponding author:

murali.ums@gmail.com ( M. Mariappan )

Published online at http://journal.sapub.org/ajbe

Copyright (C) 2011 Scientific \& Academic Publishing. All Rights Reserved prostheses small electric motors are used as actuators[3]. In myoelectric prosthetic hands these actuators are used to control number of degree of freedoms (DOFs). According to the International Federation for the Theory of Machines and Mechanisms (IFToMM) terminology[4], the degree of freedom (DOF) of a mechanism is the number of independent generalized coordinates required to define completely the configuration of a system at any instant of time[5,6].

The design of advanced robotic systems is moving from the "classical" concept of precise and stiff structures, often heavy and very complex like Robonaut Hand[7], and DLR Hand II $[8,9,10]$ to that of light and flexible ones like Cyber Hand[11], RTR II[12], RTR III[13] and Manus Hand[14] with the perspectives of increased performances, high mechanical simplification and consistent cost reduction[15]. Underactuated mechanisms (UAM) are mechanical systems with fewer control inputs (actuators) than degrees of freedoms (DOFs).

Different types of underactuated mechanisms are described by different authors but it was properly classified by He Bin et al.[5] into 4 subtypes i.e. Differential, Compliant, Triggered and Passive Mechanism. In underactuated mechanism, principle of actuation[16] is used to reduce the control complexity and to imitate grasping behavior of human hand. Challenges towards the development of a real underactuated myoelectric hand includes: the use of neural control signals together with the extraction of intent, the simple mechatronic implementation[17] having less number 
of actuators than the degree of freedoms, better functionality, light weight and cosmetic appearance to increase the personification of the hand.

In order to achieve all the requirements (targets) underactuated mechanism contributes a lot. Although classification of under-actuated mechanism based on mechanism is available but no one has given weightage to the relation of actuators with number of degree of freedoms in hand. In this paper the main focus is to study various available known prosthetic hands and then calculate a new factor termed as "CoUAM" for each of them and then to find the effectiveness of CoUAM by studying different known approaches which has been used to minimize the number of actuators.

The dexterous manipulation prosthetic hand should have all the degree of freedoms just like real human hand. Although lot of effort in the past has been carried out to make the prosthetic hand near to the human hand but still there is a huge gap to achieve that target. In past, different approaches are taken to increase the number of DOFs and to reduce the number of actuators by considering the cost, controllability, cosmetic appearance, size and weight of the prosthetic hand. Some use one motor to control one DOF (which is not an underactuated mechanism), whereas in some approaches one motor is used to control multi DOFs. In TUAT/Karlsruhe Humanoid Hand[18] only one motor is used for the whole hand. Thus different approaches are there for the underactuated mechanism but to find some factor by which a better technique of using the number of motors to DOFs is calculated. By considering different known approaches which already has been carried out for underactuated mechanisms, the calculation will be compared with the real human hand.

In section 2 of this paper, the part of the actuators in hand prostheses and the comparison of the actuators with the biological muscle discussed. Section 3 explains the optimization criteria in various studies, a novel factor CoUAM and the division of studies in 3 different zones (i.e. Red, Yellow $\&$ Green Zone) on the basis of CoUAM value. Section 4 gives the clinical correlation and section 5 brings out the conclusion.

\section{Part of Actuators in Hand Prostheses}

Actuators are the key components in any prosthetic device, one have to be very careful in their selection. Traditional actuators cannot fulfill the requirements because of their heavy weight, low power efficiency, large size and impermanency. On the other hand the design of prosthetic hand implies a series of challenging requirements to be fulfilled. Reducing number of actuators and obtain humanoid dexterous hand[19] is one of the basic objective in the design of prosthetic hand device.

Typically the numbers of actuators are chosen without considering the muscular anatomy and their number is determined by the presence of weight or space constraint. Later by studying the different approaches and calculating "CoUAM" this research will show that those hands in which number of actuators are three or four (equal to three main nerves Radial, Ulnar and Median i.e. responsible of controlling the finger movements) are more flexible option for the hand prostheses.

In the human body, a vast number of actuators are distributed throughout the body. Even a tiny blood vessel, i.e. arteriole, has a built-in actuator. There are numerous local control loops, or autonomy, around the distributed actuators in the body to fulfill the requirement of human level dexterity. The number of skeletal muscles is also huge (in human body), creating a great degree of flexibility and dexterity in its bodily motion. This allows humans and other biological creatures to develop multifunctional behaviors and perform a rich variety of dexterous tasks[20]. Motor nerves like radial, ulnar or median nerve control the group of muscles in hand responsible for different DOF. Therefore these groups of muscles may be labeled as Median, Ulnar or Radial nerve actuators respectively. These preferably should be controlled by one motor for each group of muscles supplied by isolated nerve. Later this research will show that by using 3 or 4 motors better results are obtained.

Hence to get dexterous manipulation, the actuation mechanism of actuators should resemble the biological muscle and they should also have low power efficiency. Performance of actuator depends on their power to weight ratio[21]. "Higher be the ratio better will be the actuation mechanism" (i.e. near to the biological muscle) and vice versa. Underactuated mechanism is described by many authors but it was not made objective by mathematical means, therefore it is important for all but subjective for everyone. Hence such an effort is made to make it more objective by the new concept of "CoUAM" as described in section 3 .

\section{Optimization Criterion in Various Studies}

Numerous studies have been conducted over the last few decades to achieve a balance between the aesthetic appearance and functionality of the prosthetic hand. Every one contributed a lot to make their prosthetic device as close as possible to the real human hand, although their contribution is remarkable and efforts of every one placed a benchmark for the coming prosthetic devices but still there is a huge gap that has to be filled to make prosthetic hand close to the human hand. Optimization criteria in various studies have been reviewed and to make it more understandable, these studies have been divided in three zones: red, yellow and green zone.

\subsection{Red Zone Studies}

By looking at the red zone in Table, it is evident that the studies included in this zone are unable to fulfill the requirement of the humanoid robotics. Because of their high CoUAM value, bulky and complex designs, complex controllability etc. these designs are unable to fulfill the basic 
requirement of the user, although they are highly dexterous and can easily manipulate various tasks but not the best prosthetic option for the user due to one or more reason.

Gifu Hand II[22] as shown in Figure 1, has a high potential to perform the dexterous object manipulations like the human hand, it has five fingers with 16 DOFs which are actuated by 16 servo motors. This hand can perform various tasks dexterously but had a complex control system. Size of the fingers in Gifu Hand II is same as that of the human finger. Due to large number of motors and several sensors the weight of the hand is heavy. Later from the calculation of CoUAM, it will be proved that this hand is not a good option for the amputee.

Naist Hand[23] is a four fingered hand and each finger has 3 DOFs. Separate motors are used to drive each DOF. Hence total of 12 motors are used to drive 12 DOFs. Before the development of the Naist Hand, multi DOF robot hand was developed but the problem with them is that wire driven mechanism is adopted for these hands, which requires a huge actuator unit, thus maintainability is not good for tension control of the wires.

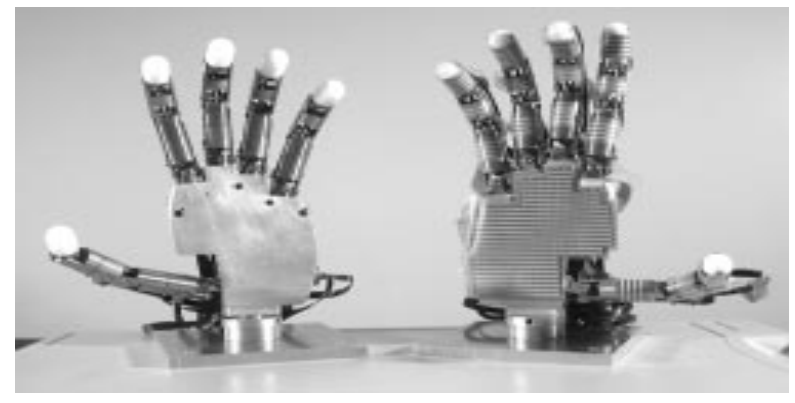

Figure 1. Gifu Hand II.

Later Naist Hand with vision-based tactile sensors solved this problem which uses gears link mechanism. But still the requirement of humanoid robotics is not fulfilled because of the high cost, heavy weight etc. Although this hand can perform various tasks dexterously but numbers of actuators are very high and no under-actuated mechanism is used.

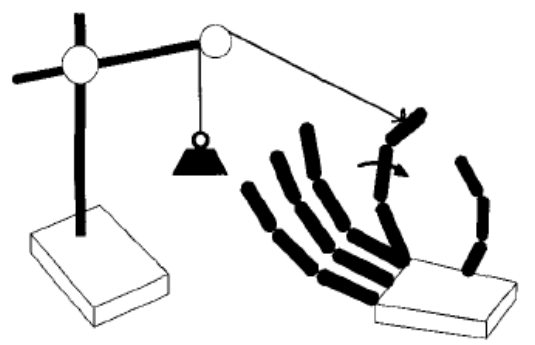

Figure 2. LMS Hand.

In traditional artificial hands, multiple degrees of freedoms (DOFs) of motion is obtained by using the several actuators which consequently the arm becomes too large and heavy weight and thus it will be a burden for users. One of the examples of such hand is the LMS Hand[24] as shown in Figure 2, with four fingers and sixteen DOFs. It can manipulate various objects with finger tips by using force and position controllers. Each finger is capable to control an applied force and react consequently. Numbers of motors used in this hand for the actuation of 16 DOFs are 16, which is very high and make the hand less anthropomorphic with complex controllability and bulky weight.

Similarly the BUAA Hand[25], which is highly dexterous with 4 fingers and 16 DOFs can perform various tasks dexterously but the drawback is the cosmetic appearance and weight of the hand (about $1.4 \mathrm{~kg}$ ) due to several actuators and sensors.

\subsection{Yellow Zone Studies}

Studies included in this zone are better than those of red zone studies. The reason for this is the adoption of underactuated mechanism to carry out simple tasks and simple grasping options. The main requirements to be fulfilled for the amputee is not just the dexterous manipulation of task (as in red zone) but also the aesthetic appearance to minimize psychological burden on the user, simple controllability, less weight, less cost and high power efficiency. For this zone, four studies have been selected.

GCUA Hand[26], a five fingered hand in which 15 DOFs are actuated by using 10 motors (actuators) using the concept of underactuated mechanism. This hand is designed to reduce the complex controllability of dexterous hands and to improve the dexterity of traditional underactuated hands. The only problem with this hand is the size, which is double than the adult's hand. In Table 1, it is found that value of CoUAM for this hand is 0.667 which is less than the value of CoUAM of every study in red zone. In 2002, TUAT/Karlsruhe Humanoid Hand[18] with five fingers and 20 DOFs is developed, which is driven by only one actuator. This hand can grasp the objects self adaptively according to the shape of the object. Since only one motor is used to carry out 20 DOFs, hand controllability is very simple and its weight is very light but the disadvantage is that it is unable to perform various daily routine tasks. To avoid the complex controllability, bulky size and psychological burden on the user, in 2006, a five fingered anthropomorphic prosthetic hand was developed and named as FIU Hand[27] with 14 DOFs which is actuated by 11 motors. Planetary gears, nuts and lead screws of identical types are used to substantially reduce the cost of the hand. This hand is fully capable of performing the daily activities of life.

\subsection{Green Zone Studies}

To reduce the complexity of the multi-fingered hand, underactuation or the motion coupling mechanism is used to reduce the number of motors without reducing the number of degrees of freedoms (DOFs). Studies included in this zone not only using the same concept of underactuated mechanism as in yellow zone, but also number of motors used to carry out several degree of freedoms. These studies very near to the human hand actuation units we will discuss in section 4. Loredana Z. et al.[28] in 2007 developed a three fingered optimized anthropomorphic hand to obtain the human like kinematics and dynamics. Hand has the capability to mimic 
the natural motion of human finger, light weight (of about $250 \mathrm{gm}$ ). Numbers of DOFs are not very high but still it gives the good results by using the concept of self adaptation. As compare to the other studies in this zone, Loredana $\mathrm{Z}$. et al. Hand is not very versatile. Whereas HIT/DLR Hand[29] is very promising among the various prosthetic options due to its handsome appearance, strong capability of grasping complex shape objects and an additional feature of thumb movement in 3-D space. For the actuation of 13 DOFs only 3 motors are being used in this design, which makes it anthropomorphic, light weight (of about $500 \mathrm{gm}$ ) and versatile.

In 2002, a three fingered underactuated prosthetic hand RTR II[30] designed with 8 DOF's. Only two motors are used one for the flexion/extension of all the fingers (including thumb) and the other for the abduction/adduction movement of the thumb. RTR II hand has high grasping functionality as compared to other commercial prosthetic devices available at that time. Actuation is intrinsic and simple control algorithm is used. Jingdong Z. et al. in 2006[31] proposed a five fingered prosthetic hand that is controlled by EMG signals.

Table 1. CoUAM(Ratio \& \%).

\begin{tabular}{|c|c|c|c|}
\hline \multirow{3}{*}{$\begin{array}{l}\text { Type of } \\
\text { Myoelectric } \\
\text { Hands }\end{array}$} & \multicolumn{2}{|c|}{ CoUAM } & \multirow{3}{*}{$\begin{array}{c}\text { Advantages } \\
\& \\
\text { Disadvantages }\end{array}$} \\
\hline & $\operatorname{Ratio}(\gamma)$ & 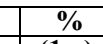 & \\
\hline & $\alpha / \beta$ & $\begin{array}{l}(1-\gamma) \\
* 100\end{array}$ & \\
\hline $\begin{array}{l}\text { Gifu hand II } \\
5 \text { fingered }\end{array}$ & $\begin{array}{l}\alpha=16 \\
\gamma=1\end{array}$ & $\begin{array}{l}\beta=16 \\
\%=0\end{array}$ & $\begin{array}{l}\text { Dexterous object manipula- } \\
\text { tions. Complex Control }\end{array}$ \\
\hline $\begin{array}{l}\text { NAIST } \\
\text { Hand }\end{array}$ & $\begin{array}{l}\alpha=12 \\
\gamma=1\end{array}$ & $\begin{array}{l}\beta=12 \\
\%=0\end{array}$ & $\begin{array}{l}\text { Changeable finger posture } \\
\text { Vision based highly accurate } \\
\text { sensing }\end{array}$ \\
\hline LMS hand & $\begin{array}{l}\alpha=16 \\
\gamma=1\end{array}$ & $\begin{array}{l}\beta=16 \\
\%=0\end{array}$ & $\begin{array}{l}\text { Fine finger control } \\
\text { Manipulation by finger tips } \\
\text { Elastic cables used for mani- } \\
\text { pulation }\end{array}$ \\
\hline BUAA Hand & $\begin{array}{l}\alpha=16 \\
\gamma=1\end{array}$ & $\begin{array}{l}\beta=16 \\
\%=0\end{array}$ & $\begin{array}{l}\text { Highly dexterous Non anth- } \\
\text { ropomorphic. Can be used as } \\
\text { test bed for future research }\end{array}$ \\
\hline $\begin{array}{l}\text { GCUA Hand } \\
5 \text { fingered }\end{array}$ & $\begin{array}{l}\alpha=10 \\
\gamma=0.667\end{array}$ & $\begin{array}{l}\beta=15 \\
\%=33\end{array}$ & $\begin{array}{l}\text { Self- adaptive grasping } \\
\text { Nice, valid \& low cost but size } \\
\text { is double than adult's hand. }\end{array}$ \\
\hline $\begin{array}{l}\text { TUAT/Karls } \\
\text { ruhe Hand } \\
5 \text { fingered }\end{array}$ & $\begin{array}{l}\alpha=1 \\
\gamma=0.050\end{array}$ & $\begin{aligned} \beta & =20 \\
\% & =95\end{aligned}$ & $\begin{array}{l}\text { Human like manipulation } \\
\text { abilities. Light weight } \\
\text { Simple control system \& } \\
\text { Self-adaptive grasp. }\end{array}$ \\
\hline $\begin{array}{l}\text { FIU Hand } \\
5 \text { fingered }\end{array}$ & $\begin{array}{l}\alpha=11 \\
\gamma=0.72\end{array}$ & $\begin{aligned} \beta & =14 \\
\% & =28\end{aligned}$ & $\begin{array}{l}\text { Ability to pick up small ob- } \\
\text { jects. Aesthetic, Cheaper, Less } \\
\text { complex control. Light Weight }\end{array}$ \\
\hline $\begin{array}{l}\text { TH-I Hand } \\
5 \text { fingered }\end{array}$ & $\begin{array}{l}\alpha=3 \\
\gamma=?\end{array}$ & $\begin{array}{l}\beta=? \\
\%=?\end{array}$ & $\begin{array}{l}\text { Light weight with fewer DOFs } \\
\& \text { simple controllability. }\end{array}$ \\
\hline $\begin{array}{l}\text { Loredana } Z . \\
\text { et al. Hand } \\
3 \text { fingered }\end{array}$ & $\begin{array}{l}\alpha=4 \\
\gamma=0.445\end{array}$ & $\begin{array}{c}\beta=9 \\
\%=55.5\end{array}$ & $\begin{array}{l}\text { Light weight }(250 \mathrm{gm}) \\
\text { Self -adaptive grasp } \\
\text { Neglected presence of friction }\end{array}$ \\
\hline $\begin{array}{l}\text { HIT/DLR } \\
\text { Hand } \\
5 \text { fingered }\end{array}$ & $\begin{array}{l}\alpha=3 \\
\gamma=0.317\end{array}$ & $\begin{array}{c}\beta=13 \\
\%=68.3\end{array}$ & $\begin{array}{l}\text { Light weight (Wt. 500g) \& } \\
\text { simple constructed multi DOF } \\
\text { hand. Perform nips, grasps \& } \\
\text { complex shapes. Aesthetic }\end{array}$ \\
\hline $\begin{array}{l}\text { RTR II } \\
\text { Hand } \\
3 \text { fingered }\end{array}$ & $\begin{array}{l}\alpha=2 \\
\gamma=0.250\end{array}$ & $\begin{aligned} \beta & =8 \\
\% & =75\end{aligned}$ & $\begin{array}{l}\text { Self-adaptive grasp. } \\
\text { Intrinsic actuation } \\
\text { Simple control algorithm. }\end{array}$ \\
\hline $\begin{array}{l}\text { Jingdong } Z \text {. } \\
\text { et al. Hand } \\
5 \text { fingered }\end{array}$ & $\begin{array}{l}\alpha=3 \\
\gamma=0.214 \\
6\end{array}$ & $\begin{aligned} \beta & =14 \\
\% & =78 .\end{aligned}$ & $\begin{array}{l}\text { Shape adaptation, Multiple } \\
\text { grasping options } \\
\text { Low cost, light weight, user } \\
\text { friendly.\& Less dexterity }\end{array}$ \\
\hline $\begin{array}{l}\text { AR hand III } \\
5 \text { fingered }\end{array}$ & $\begin{array}{l}\alpha=3 \\
\gamma=0.214\end{array}$ & $\begin{array}{c}\beta=14 \\
\%=78.6\end{array}$ & $\begin{array}{l}\text { Spherical, cylindrical, preci- } \\
\text { sion and lateral grasp, light } \\
\text { weight, dexterous }\end{array}$ \\
\hline
\end{tabular}

Three fingers are active and other two fingers are passive and by using only few stepper motors (actuators) in his design several grasping options are possible. So it is user friendly, low cost, light weight $(0.55 \mathrm{~kg})$ and automatically adopt the shape of different objects.

D. Yang et al. in 2009 developed a five fingered myoelectric prosthetic hand named as AR hand III[13]. This newly designed robot hand (i.e. controlled by surface EMG) cannot have too many actuators, since EMG signals highly influenced by noise, sweat and muscle fatigue. It can successfully operate different various objects with different sizes.

Significance of coefficient of underactuated mechanism "CoUAM" is the "ratio between numbers of motors to the total number of degrees of freedom (DOFs)" but main objectives in the design of any humanoid hand are to get maximum number of degree of freedom by using minimum number of actuators, to avoid complex controllability, large size, heavy weight, unaesthetic appearance and psychological burden on user etc. Although lot of efforts in the past have been carried out to make prosthetic hand as close as possible to the real human hand and to make it more acceptable but still there is a huge gap between artificial hand and the real human hand which is highly dexterous and can manipulate various tasks easily and self adaptive. One of the simple techniques that maintain the humanistic appearance, size and weight of an artificial hand without scarifying its DOFs is by adopting the underactuated mechanism approach[32]. In the underactuated mechanism electric motors are replaced by passive elements such as spring and mechanical limits. Since underactuated mechanism is based on minimum number of motors to get the maximum number of degree of freedoms (DOFs), still there is no study available to make it more objective. Hence target of this research is to calculate factor named as CoUAM for some of the available studies and then compare it with the real hand's CoUAM which is defined as the ratio between the number of nerve unit (considered as actuator) to the total degree of freedom available.

Table 1 is divided into three zones: Red, Yellow and Green Zone. Division in three different colours basically shows the nearness of the prosthetic hand device to the real human hand. Red colour shows that the prosthetic hand is quite far from the real hand the reason for this is "very high value of CoUAM" (i.e. maximum or equal to 1) with many actuators. It may be highly dexterous but on the other hand it has complex controllability, unaesthetic appearance, heavy weight and high cost. So the available prosthetic hands in this zone are not considered to be good option for the amputees. Whereas in the yellow zone although it is better as compared to red zone but still unable to meet the requirement of humanoid robotics. It may be very light/heavy weight depending upon the number of actuators used. With minimum number of actuators (i.e. equal to 1 ) it can be aesthetic with simple controllability but on the other hand is unable to perform daily routine tasks and with the maximum number of actuators (i.e. equal to 10 in that zone) it may be dexterous but with complex controllability, big size and heavy weight makes it unable to fulfill the requirements of the users. 
However the green zone in the Table 1 shows the maximum value of CoUAM to be 0.445 and its minimum value is 0.214 which is not very far from the ideal value of CoUAM (i.e. 0.143 i.e. $3 / 21$ ). Upon analyzing the advantages/disadvantages, size, weight, cosmetic appearance, controllability, functionality perhaps in every aspect it is quite clear that the studies included in this zone shows a much better results.

\section{Clinical Correlation}

All the hand movements are controlled by 3 nerves i.e. Median, Ulnar and Radial. By applying the same principle, it is presumed that all the 21 DOFs preferably should be controlled by 3 motors (ideally in any electrical hand). Therefore, an ideal hand CoUAM will be $3 / 21(1 / 7)=0.143$. If any hand system with a CoUAM of $0.143(86 \%)$ or closer with a good functional similarity, it will be considered as an ideal hand. Higher the ratio either by increasing the number of motors or less no's of DOFs will result in poor quality of hand. Our current ongoing project titled "Technology Advancement in Nerve Guided Actuators and Normalizers (TANGAN) in Myoelectric Hand" is based on anatomical and functional similarity in anthropomorphic hand in terms of number of phalanges, joints, nerves, tendons, different pulley and DOF. Therefore it is possible to be having a stimulant of $1 / 7$ of the hand in terms of morphology (cosmetics) and physiology (function).

Muscles in the human body and motors in myoelectric hands are considered as actuators. Muscles are controlled by nerves and servo motors are moved by rechargeable batteries connected with cables. For the sake of simplicity, a group of muscles supplied by one nerve like ulnar nerve is considered as Ulnar Actuator Motor Unit (UAMU). Similarly other two nerves in hand will constitute the Radial Actuator Motor Unit (RAMU) and Median Actuator Motor Unit (MAMU). Keeping in view of a single muscle i.e. abductor pollicis longus muscle, innervated by radial nerve consists of 400-500 motor unit and all these 3 nerves of hands supply multiple muscles would result into thousands of motor units. Ulnar nerve supply all interossei muscles ( 8 in numbers) might constitute thousands of motor unit. Therefore motor unit should not be mixed with Actuators Motor Units.

In anatomy of the hand, every nerve (i.e. radial or median or ulnar) is responsible for the actuation of multi muscles or in other words each of them is responsible for multi DOFs. By using the same principle, review of available studies, the proposed novel concept of coefficient of UAM which avoids complex controllability, unaesthetic appearance, heavy weight, and psychological burden on user, it is shows that the green zone studies are the suitable one.

In case of 21 DOFs in hand is controlled by 21 motors (actuators) then the underactuated mechanism is not realized. In contrast, if one motor will be used it will be highly underactuated but its functionally may not be good. Therefore based upon " 3 nerve 3 actuator principal" from the CoUAM analysis, preferable number of motors in hand should be 3 .

\section{Conclusions}

Underactuated mechanism is based on minimum number of motors to get the maximum number of DOF's. Since no studies are available to make it more objective, the novel approach for the classification of underactuated mechanism CoUAM gives a new thought to the researchers to fabricate such kind of myoelectric prosthetic hands for the amputees which fulfills the requirements of humanoid robotics.

The CoUAM of randomly selected studies is calculated separately. On the basis of its value and by analyzing the various optimization criterion, their merits/demerits, cost, weight, cosmetic appearance, ease of controllability and their functionality, these studies are divided in Red, Green and Yellow Zones. Division in three zones is not only on the basis of the above factors but also by the clinical correlation and it is found that the studies included in the Green Zone gives a very promising results (i.e. they are quite close to the real human hand).

\section{REFERENCES}

[1] Iftikhar, M., Jan, S. M. M., and Mariappan, M. 2011. COEFFECIENT OF UNDERACTUATED MECHANISM (CoUAM) Novel Approach for Underactuated Mechanism of Myoelectric Hand Prosthesis. The 5th International Conference on Bioinformatics and Biomedical Engineering (iCBBE 2011) (Wuhan, China, May 10-12, 2011)

[2] Atkins, D. J., Heard, D. C. Y., Donovan, W. H., 1996. Epidemiologic Overview of Individuals with Upper-Limb Loss and Their Reported Research Priorities. J. of Prosthetics and Orthotics. 8, 1, 2-11

[3] DOI=http://en.wikipedia.org/wiki/Actuator

[4] Dellon, B., and Matsuoka, Y. 2007. Prosthetics, exoskeletons, and rehabilitation. IEEE Robotics and Automation magazine. 14, 1 (Mar. 2007)

[5] Bin, H., Zhen, L. W., and Fen, L. H. 2010. A Survey on Underactuated Mechanism. In Proc. of 2nd International Conference on advanced Computer Control (ICACC) (Shenyang, Mar. 27-29 2010). 4, $551-555$.

doi:10.1109/ICACC.2010.5486858

[6] IFToMM 1991. Terminology for the theory of machines and mechanisms. Mechanism and Machine Theory (1991). 26, 435-453

[7] Ambrose R. O., Aldridge H., Askew, R. S., Burridge, R. R., Bluethmann, W., Diftler, M., Lovchik, C., D. Magruder, D., and Rehnmark, F. Robonaut: NASA's space humanoid. IEEE Intelligent Systems and their Applications (2000). 15, 57-63

[8] "Butterfass, J., Grebenstein, M., Liu H., and Hirzinger, G. DLR-Hand II: Next generation of a dexterous robot hand. Proceedings of IEEE International Conference on Robotics and Automation (Seoul, Korea, 2001). 1, 109-114 
[9] A. Karnik Biagiotti, L., Lotti, F., Melchiorri, C., and Vassura, G. How Far is the Human Hand? A Review on Anthropomorphic Robotic End-Effectors. University of Bologna, Italy, 2002,[2009-4-5],

DOI=http://www-lar.deis.unibo.it/woda/data/deis-lar-publica tions/3cbd.Document.pdf of Science, Bangalore, India, Jan. 1999

[10] Bicchi, A. Hands for dexterous manipulation and robust grasping: A difficult road toward simplicity. IEEE Transaction on Robotics and Automation (2000), 16, 652-662

[11] Zollo, L., Roccella, S., Guglielmelli, E., Carrozza, M. C., and Dario, P. Biomechatronic design and control of an anthropomorphic artificial hand for prosthetic and robotic applications. IEEE/ASME Transactions on Mechatronics (2007). 12, $418-429$

[12] Carrozza, M. C., Cappiello, G., Cavallaro, E., Mieera, S., Vecchi, F., and Dario, P. Design and control of an underactuated cybernetic artificial hand. Proceedings of 2004 World Automation Congress (Seville, Spain, 2004). 1, 111-116

[13] Yang, D.-peng, Zhao, J.-dong, Gu, Y.-kun, Wang, X.-qing, Li, N., Jiang, L., et al. (2009). An Anthropomorphic Robot Hand Developed Based on Underactuated Mechanism and Controlled by EMG Signals. Journal of Bionic Engineering. 6, 3, 255-263. Jilin University.

Doi: 10.1016/S1672-6529(08)60119-5

[14] Pons, J. L., Rocon, E., and Ceres, R., 2004. The MANUS-HAND dexterous robotics upper limb prosthesis: Mechanical and manipulation aspects. Autonomous Robots (2004). 16, 143-163

[15] Biagiotti, L., Lotti, F., Melchiorri, C., and Vassura, G., 2003. Mechatronic design of innovative fingers for anthropomorphic robot hands. Proceedings. ICRA'03. IEEE International Conference on Robotics and Automation. 3, 3187-3192. DOI:http://ieeexplore.ieee.org/xpls/abs all.jsp?arnumber $=12$ 42081

[16] Laliberte, T., and Gosselin, C. Simulation and design of under-actuated mechanical hands. Mechanism and Machine Theory (1998). 33, 39-57

[17] Jonathon, S., Paul, F. P., and Todd, K. The future of artificial limbs.

DOI:http://www.bordeninstitute.arm.mil/published_volumes /amputee/CCAchapter27.pdf

[18] Fukaya, N., Toyama, S., Asfour, T., and Dillmann, R. Design of the tuat/karlsruhe humanoid hand. Intelligent Robots and Systems, 2000.(IROS 2000). Proceedings. 2000 IEEE/RSJ International Conference on, IEEE, 2002. 1754-1759

[19] Malhotra, M., and Matsuoka, Y. The Relationship between Actuator Reduction and Controllability for a Robotic Hand. (Apr. 9 2010). Computer.

DOI:http://neurobotics.cs.washington.edu/papers/BioRob201 0/Malhotra BioRob2010.pdf

[20] DOI:http://biorobotics.snu.ac.kr/papers/16\%20Design\%20an d $\% 20$ Control $\% 20$ of $\% 20$ Vast $\% 20$ DOF $\% 20$ Wet $\% 20$ SMA $\% 2$ 0 Array\%20Actuators.pdf

[21] Mascara, S., and Asada, H. Wet shape memory alloy actuators for active vasculated robotic flesh. In Proceedings of
2003 IEEE Zntemational Conference on Robotics and automation (Sept. 14-19 2003). 2.

doi:10.1109/ROBOT.2003.1241814

[22] Kawasaki, H., Komatsu, T., and Uchiyama, K. Dexterous anthropomorphic robot hand with distributed tactile sensor: Gifu hand II. IEEE/ASME Trans. Mechatronics (September 2002). 7, 3, 296-303

[23] Ueda, J., Ishida, Y., Kondo M., and Ogasawara, T. Development of the NAIST-Hand with Vision-based Tactile Fingertip Sensor. Proceedings of the 2005 IEEE International Conference on Robotics and Automation (Barcelona, Spain). 2332-2337

[24] Gazeau, J. P., Zehloul, S., Arsicault, M., and Lallemand, J. P. 2001. The LMS hand: force and position controls in the aim of the fine manipulation of objects. Proceedings 2001 ICRA. IEEE International Conference on Robotics and Automation (Seoul, Korea). 2642-2648. (Cat. No.01CH37164). doi: 10.1109/ROBOT.2001.933021

[25] Zhang, Y., Han, Z., Zhang, H., Shang, X., Wang, T., and Guo, W. Design and Control of the BUAA Four-Fingered Hand. In Proc. of the 2001 IEEE International Conference on Robotics and Automation (Seoul, Korea May 21-26, 2001). 2517-2522

[26] Zhang, W., Che, D., Chen, Q., and Du, D, A Dexterous and Self-adaptive Humanoid Robot Hand: Gesture-Changeable Under-Actuated Hand. International Conference on Intelligent Robotics and Applications (Singapore): Springer. $515-525$

[27] Nasser, S., Rincon, D., and Rodriguez, M. Design of an anthropomorphic underactuated hand prosthesis with passive-adaptive grasping capabilities. Conference on Recent Advances in Robotics (Florida 2006). 1-7

[28] Loredana, Z., Stefano, R., Gugliemelli, M. C. C., and Dario, P. 2007. Biomechatronic Design and Control of an Anthropomorphic Artificial Hand for Prosthetic and Robotic Applications. IEEE/ASME Transactions on Mechatronics. 13, 4, 741-429. doi: 10.1109/TMECH.2007.901936

[29] Huang, H., Jiang, L., Zhao, D. W., Zhao, J. D., Cai, H. G., Liu, H., Meusel, P., Willberg, B., and Hirzinger, G. 2007. The development on a new biomechatronic prosthetic hand based on under-actuated mechanism. IEEE/RSJ International Conference on Intelligent Robots and Systems (Beijing, China). 3791-3796.

DOI:http://ieeexplore.ieee.org/xpls/abs_all.jsp?arnumbers $=4$ 058996

[30] Massa, B., Roccella, S., Carrozza, M. C., and Dario, P. Design and development of an underactuated prosthetic hand. Proceedings of the 2002 IEEE International Conference on Robotics \& Automation. 3374-3379

[31] Zhao, J., Jiang, L., Shi, S., Cai, H., Liu, H., and Hirzinger, G. A Five-fingered Underactuated Prosthetic Hand System. 2006 International Conference on Mechatronics and Automation (Luoyang, China, 2006). 1453-1458

[32] Azlan, N.Z., Yamaura, H. 2010. Underactuated Anthropomorphic Finger Mechanism for Grasping and Pinching with Optimized Parameter. Journal of Computer Science. 6, 8, 928-933 